\title{
REFLEXIONS SUR QUELQUES MÉTHODES DE MESURES \\ D'ÉTOILES DOUBLES ET LEUR EMPLOI
}

\author{
P. LACROUTE \\ Observatoire de Strasbourg, France
}

J'ai été conduit à m'intéresser à la mesure des étoiles doubles au cours d'un projet de mesures spatiales de positions d'étoiles par une méthode de balayage photoélectrique. L'existence d'étoiles doubles perturbe les mesures. J'ai donc étudié les limitations imposées aux mesures de position par balayage d'images du fait de composantes d'étoiles doubles. Cette étude m'a donné l'occasion de réfléchir au problème de la mesure des étoiles doubles et voici le résultat de mes réflexions.

Les conclusions du rapport de $\mathrm{M}$. Couteau montrent le grand intérêt qu'il y a à faire des mesures sur des étoiles serrées pour avoir plus de programmes possibles aboutissant à des orbites calculables. Ces conclusions doivent être encore renforcées si on considère que pour les études d'évolution ce sont en général les orbites à plus courte période qui doivent être les plus intéressantes.

On peut faire une étude de la précision possible si on faisait des mesures dans l'espace avec un instrument stable et parfait, en étudiant complètement les images, les seules limites étant imposées par la diffraction et par la magnitude en raison de la structure granulaire de la lumière. L'incertitude limite $\varepsilon$ est proportionnelle à $1 / D(D$ étant le diamètre d'ouverture de l'instrument) par suite de l'amélioration de la finesse des images, et à $1 /\left(\sqrt{D^{2} E T}\right.$ ) ( $E$ étant l'éclat des étoiles, $T$ la durée d'accumulation des informations) par suite de l'augmentation des informations reçues. On a donc

$$
\varepsilon \sim D^{2} \sqrt{E T}
$$

Mais de telles mesures dans l'espace ne paraissent pas devoir être immédiates et seront de toutes façons très couteuses. Il convient donc d'examiner ce qu'on peut faire au sol pour se rapprocher de cet idéal.

$\mathrm{Au} \mathrm{sol} \mathrm{les} \mathrm{résultats} \mathrm{pratiques} \mathrm{ne} \mathrm{peuvent} \mathrm{pas} \mathrm{être} \mathrm{aussi} \mathrm{bons,} \mathrm{essentiellement} \mathrm{parce}$ que les images sont déformées et déplacées par l'atmosphère, mais aussi parce que nous recevons moins de photons et parce que l'instrument n'est jamais parfait.

Pour approcher la précision limite il faudrait, en outre, employer des méthodes qui utilisent complètement les informations apportées par les photons reçus. On obtiendra les résultats les plus précis en faisant concourrir toute les informations reçues sur l'image pour la comparer à l'image parfaite de structure connue. Si les images sont mal séparées l'analyse plus difficile de l'image fait perdre de la précision.

Il serait donc bon en étudiant de nouvelles méthodes d'avoir constamment présent à l'esprit ce que serait l'exploitation idéale des informations que les photons nous apportent et d'examiner dans quelle mesure la méthode en question elle-même fait 
perdre des informations qui auraient pu contribuer à déterminer les positions relatives et les $\Delta m$. Par ailleurs il faudrait étudier pratiquement comment, en fait, l'atmosphère diminue le rendement de principe de la méthode et ajuster la méthode pour réduire au mieux cette perte de rendement.

Ainsi la méthode de photographie électronique signalée par $\mathrm{M}$. Laques recueille la totalité des informations que les photons passant à travers l'atmosphère sont susceptibles de donner. Il faudrait lui associer une méthode d'exploitation des plaques ne faisant rien perdre de ces informations. Il serait souhaitable que cette méthode d'exploitation soit assez rapide. Par ailleurs il faudrait sans doute, après étude, diviser la pose totale pour réduire l'étalement des images dû à l'atmosphère.

Les méthode de balayage d'images expérimentées par le Dr Franz et par Høg ont l'inconvénient de n'utiliser chaque partie des images que pendant une fraction du temps assez faible, d'où perte d'information. En outre le balayage successif dans deux sens fait perdre un facteur 2 sur le temps. Mais les informations recueillies sont sous une forme extrêmement commode pour être analysées à fond par le calcul. En outre, on peut montrer qu'on ne perd rien sur la mesure des positions relatives et des $\Delta m$ en étudiant deux profils de l'image au lieu de l'image à deux dimensions. L'analyse des profils devrait probablement donner ses meilleurs résultats en recherchant les décalages qui associent au mieux des profils successifs, puis en cherchant la meilleure représentation du profil global par la somme de deux profils identiques à déterminer, ainsi que leur séparation et leur $\Delta m$.

Toutes les études en cours, très prometteuses, permettront de connaître les limites de ce qu'on peut faire au sol et ce sera extrêmement précieux. Cela ne veut pas dire que les nouvelles méthodes mises au point remplaceront complètement à l'avenir les méthodes anciennes, œil ou photographie classique parce que les nouvelles méthodes seront probablement beaucoup plus lourdes. Mais il est indispensable de savoir ce qu'on peut obtenir au sol par chaque méthode et le prix qu'il faut y mettre. II sera alors possible d'établir les programmes les plus judicieux.

\section{Discussion}

Laques: En rapport avec ce que dit le Professeur Lacroute, concernant l'analyse fine des images de diffraction, je voudrais montrer une analyse isodensitométrique d'image d'étoile double qui montre la présence des détails.

Lacroute: Il est très possible qu'en étudiant de très près ces structures, vous puissiez avoir aussi bien sur le $\Delta m$ que sur l'écart des mesures particulièrement bonnes. Enfin dans mon esprit le plus intéressant serait d'étudier les étoiles mal séparées si possible.

Rösch: La méthode de balayage donne une simple infinité d'informations, la caméra électronique en donne une double infinité, mais elle est plus coûteuse à l'exploitation. Il faut la réserver aux cas difficiles.

Lacroute: La comparaison des deux méthodes paraît judicieuse. Les méthodes de balayage travaillent sur une direction, donnent une information moins fine, mais par contre elles ont l'avantage d'être stockées dans des calculateurs et exploitées de façon très systématique. Je suis convaincu que l'avancement des travaux du Dr Franz et du Dr Høg doit permettre d'étudier ces problèmes de façon très systématique et intéressante afin de connaître nos limites.

Hog: Will the average scan in any direction be symmetric? Secondly, will the average image be rotation-symmetric? If the first answer is affirmative, it makes it easier to avoid symmetric errors in $Q$ and $\Delta m$. 
Rösch: Il n'y a pas de raison pour une dissymétrie où un balayage a une dimension, mais il n'y a effectivement pas symétrie de révolution (voir IAU Symposium No. 19, Rome, 1962).

Rakos: The electronic camera still has the problem of a non-uniform sensitivity of a photocathode. The use and the reduction procedure of such a choice is too complicated to be applied in a routine program.

Rösch: D'accord, mais on calibre en posant par points.

Laques: La remarque de M. Rakos est juste lorsque l'on veut des images différentes sur l'ensemble de la photocathode; mais l'inconvénient n'existe plus si on laisse l'image au même point de la photocathode et si, au lieu de déplacer l'image sur la photocathode on la déplaçait sur la plaque, c'est-à-dire si l'on déplaçait la plaque; c'est un problème technique qu'il faut résoudre, ce qui est difficile car la plaque est dans le vide.

Strand: The effectivity of the Kron electronographic camera is demonstrated by his photometry of $\mathrm{M} 13$ with 15 min exposures with the $61^{\prime \prime}$ astrometric reflector. He not only succeeded in measuring the faintest star in Baum's sequence obtained with the 200 in telescope which is 23,1 magnitude photographic, but he also measured an adjacent star of 23,6 magnitude. The measurements of the plate was done with the modified Joyce Loebel isodensitometer.

Franz: There is, at the present time, so much need for accurate photometries of the components of double stars that all possible methods should be used to obtain more observations. This is not the time for jealous competition. The relative merits and the compatibility of these techniques can be discussed at a later time. 